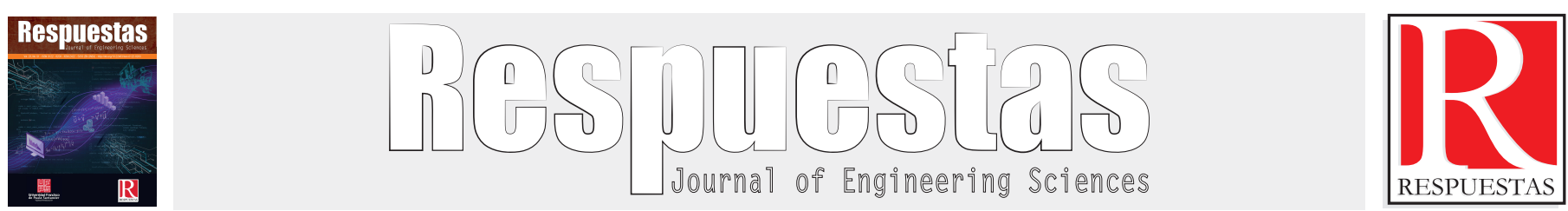

Artículo Original

https://doi.org/10.22463/0122820X.1602

\title{
Gerencia estratégica de proyectos: Aplicación del modelo de Constructibilidad
}

\author{
Strategic management of projects: Application of the Constructibility model \\ Yan Carlos Ureña-Villamizar ${ }^{\mathrm{a}}$, Jairo Alberto Cardenas ${ }^{\mathrm{b}}$, Yusbely Cecilia Castrillón-Jaimes ${ }^{\mathrm{c}^{*}}$. \\ "Postdoctor in Human Sciences, yan.urena@uniminuto.edu, orcid.org/0000-0002-9970-159X, Corporación Universitaria Minuto de Dios, Cúcuta, Colombia. \\ ${ }^{b}$ Doctor in Management, jairo.cardenas@uniminuto.edu, orcid.org/0000-0003-1816-7872, Corporación Universitaria Minuto de Dios, Cúcuta, Colombia. \\ $c^{*}$ Master in Pedagogical Practice, yusbely.castrillon@unipamplona.edu.co, orcid.org/0000-0002-6239-5883, Universidad de Pamplona, Cúcuta, Colombia.
}

Cómo citar: Y.C. Ureña-Villamizar, J.A. Cardenas y Y.C. Castrillón-Jaimes, "Gerencias estratégica de proyectos: Aplicación del modelo de Constructibilidad", Respuestas, vol. 23, no. S1, pp. 113 - 118, 2018. https://doi.org/10.22463/0122820X.1602

Recibido: Febrero 29, 2018; Aprobado: Julio 28, 2018

\begin{tabular}{ll}
\hline & RESUMEN \\
\hline Palabras Clave: & La presente indagación está dirigida a proponer estrategias gerenciales para la administración de proyectos de construcción, la \\
Constructibilidad & cual se basa en un conjunto de conocimientos y experiencias que a lo largo del tiempo ha dado resultado de gran valor en el \\
Estrategia & conocimientos y experiencia en la construcción, las operaciones de planificación, diseño y sobre el terreno para alcanzar los \\
Gerencia & objetivos generales del proyecto, en este sentido el propietario y el contratista deben estar a la par en cuanto a información en la \\
Proyectos & aplicación de la constructibilidad debido a que el contratista se enfoca más en las prioridades establecidas por el propietario y \\
& no en el impacto progresivo y efectivo del proyecto. Por cual se requirió del análisis documental y estandarización de los \\
& conocimientos que integran la gestión estratégica, como herramienta su utiliza la hoja de ruta de aplicación siendo la guía para \\
la organización, perfeccionamiento y aplicación de la constructibilidad, implementada por las empresas en los proyectos de & construcción, la cual comprende cinco etapas, entre ellas: comprometerse a implementar constructibilidad, establecer un \\
programa de constructibilidad corporativa; obtener las capacidades de constructibilidad, implementación del plan & \\
constructibilidad y actualización del programa corporativo.
\end{tabular}
constructibilidad y actualización del programa corporativo.

Keywords:

Constructability

Strategy

Management

Project

\section{ABSTRACT}

This inquiry is directed to propose management strategies to manage construction projects, which is based on a set of knowledge and experience over time has been of great value in the context dela Construction Industry to support the potential cost savings efforts, optimal use of knowledge and experience in construction, operations planning, design and field to achieve the overall project objectives in this respect the owner and the contractor should be the same information regarding the implementation of constructability because the contractor is more focused on the priorities set by the owner and not the progressive and effective impact of the project. Which was required for document analysis and standardization of knowledge that make strategic management as a tool your using the roadmap implementation being the guide for the organization, development and implementation of constructability, implemented by companies in projects construction, which comprises five stages, including: commit to implementing constructability, constructability establish a corporate program, obtain the capabilities of constructability, constructability plan implementation and updating of the corporate program.

\section{Introducción}

La gerencia de proyectos es la responsable de la dirección de los mismos además de que forma parte de la estructura funcional de las empresas o industrias; esta requiere muchas veces de una estructura que facilite las actividades además de tareas del proyecto con la finalidad de llevarlo al éxito. Ahora bien, este éxito se logra tomando en cuenta las herramientas de modelos estratégicos lo cual hace que se logren a través de una planificación de los objetivos planteados y lleva a la empresa mucho más allá de sus metas.

Por su parte, [1] en proyectos asociados a otros ámbitos ajenos a la construcción y en donde no existe la figura de la dirección facultativa (que conlleva responsabilidades técnicas y jurídicas), tales como proyectos informáticos, de lanzamientos de productos, nuevos negocios, entre otros, la gestión tiene características que se pueden asociar a un auténtico liderazgo de la operación, tanto desde el punto de vista de responsabilidad como de gestión de todos los recursos y tiene más sentido, entonces, hablar de dirección en términos amplios. El equipo que gestiona el proyecto no lo desarrolla, sino que lo gestiona, siendo el soporte tecnológico, administrativo y supervisor del cliente, y representándole, cuando así se decide, delante del proyectista, los contratistas, los organismos públicos u otros actores que puedan intervenir a lo largo del proceso. La utilización de este procedimiento de gestión como lo es el constructibilidad, es más evidente en grandes proyectos fundamentados en magnitudes elevadas en número y dispersión, con varios proyectistas y contratistas.

*Autor de correspondencia. 


\section{Modelo estratégico}

La correcta gestión de los proyectos pretende ser una inversión de rentabilidad y la metodología de los mismos no puede ser considerada como un fin en sí misma, sino como una ayuda encaminada a facilitar la consecución de los resultados. El grado de minuciosidad y desarrollo de la metodología deberá adaptarse a la dificultad así como el tamaño de cada proyecto. Por su parte, [2] el modelo para la gestión en la fase de implementación de proyectos menores es darle una guía basada en las tareas así como actividades a ejecutar poniendo en práctica cada uno de los objetivos de la organización para poder cumplir con la totalidad del proyecto.

De la misma manera, [3] señala que los modelos estratégicos "son documento oficial cuyo propósito es describir la estructura de funciones y departamentos de una organización, así como las tareas específicas y la autoridad asignada a cada miembro del organismo" (p. 86). Por su parte, Santibáñez [4], señala que el modelo de gestión no es más que una estructura realizada para la toma de decisión dentro de la organización. En otro sentido, es importante que la organización le preste atención a estos elementos para que de manera coordinada se pueda desarrollar, y se logren mejores resultados de gestión a favor del desarrollo de la empresa.

\section{Constructibilidad}

Antes de comenzar a definir teóricamente la constructibilidad es necesario señalar que es un sistema en el cual se puede conseguir de manera óptima la composición así como integración de los conocimientos además de experiencia constructivos en las operaciones de planificación, ingeniería y construcción; orientado a tratar las peculiaridades de la obra y las restricciones del entorno con la finalidad de alcanzar los objetivos del proyecto. Aunado lo anterior, la constructibilidad es una herramienta utilizada para realizar proyectos de construcción, la cual se basa en un conjunto de conocimientos y experiencias que a lo largo del tiempo ha dado resultado de gran valor.

En otras palabras para [5], la constructibilidad es el uso óptimo de todos los conocimientos de construcción y de la experiencia en planificación, diseño, procura y operaciones con el fin de alcanzar todos los objetivos del proyecto. En otras palabras, es una mezcla de los elementos de construcción con las nociones básicas de planificación con el fin de cubrir las necesidades de una empresa o industria. En otro sentido, la constructibilidad es uno de los aspectos que más claramente inciden en el control del coste y en el plazo.

\section{Hoja de ruta de aplicación de la constructibilidad}

Un proyecto que arroje soluciones no construibles o difícilmente construibles provocan un inmediato aumento del coste o el plazo, si no los dos a la vez, sobre los valores previstos; se podría decir que todo es construible en un proyecto o ejercible y que solo dependen del precio y del plazo que se le asocie. Ahora bien, en constructibilidad se utiliza una hoja de ruta la cual comprende 6 etapas: Comprometerse a implementar Constructibilidad; Establecer un programa de Constructibilidad Corporativa; Obtener las capacidades de constructibilidad; Implementación del Plan Constructibilidad; Implementar Constructibilidad y Actualización del Programa Corporativo. Dicha hoja pretende ser útil para los propietarios, diseñadores y contratistas, la cual es dirigida a programas corporativos de constructibilidad y al programa del proyecto de factibilidad de construcción.

\section{Hito de la hoja de ruta para la implementación}

Se realiza un solido programa de constructibilidad corporativo antes de iniciar un programa a nivel de proyecto, este se enfoca hacia asegurar que todos los soportes estén en su lugar cuando sea necesario, están involucrados 2 hitos en el desarrollo del programa corporativo:

\section{Hoja de ruta hito 1}

Comprometerse a la aplicación de constructibilidad: este pasó es crítico para tener éxito, el personal en todos niveles de la empresa debe estar profundamente familiarizado con los objetivos, métodos y conceptos de constructibilidad. Realizar la autoevaluación e identificar las barreras: debe llevarse acabo una evaluación de las capacidades actuales de constructibilidad y las prácticas. Los resultados de los procedimientos de auto-evaluación contribuyen a clarificar los objetivos del programa de constructibilidad, identificar los beneficios del programa actuales y futuras; definición de áreas de mejora del programa y determinar el estado actual del proceso de evaluación comparativa para futuras referencias. Existen quince parámetros significativos de proyectos corporativos para un programa de constructibilidad las cuales se dividen en cuatro grandes clasificaciones:

\section{Cultura Corporativa}

La cultura corporativa constituye la parte más compleja de una empresa debido a que tiene que ver con la naturaleza humana. [6] la define como el conjunto de valores, costumbres, hábitos y creencias existentes en una organización. La cultura se refiere a un sistema de significados compartidos por una gran parte de los miembros de una organización

\section{Personal}

Para [6], es el conjunto de personas que trabajan en un mismo organismo, empresa o entidad.

\section{Documentación y Seguimiento: Se debe tener en cuenta:}


*Documentación de los procedimientos de constructibilidad y los esfuerzos de la empresa y los niveles del proyecto.

*Los esfuerzos para capturar y comunicar las lecciones aprendidas de constructibilidad.

*El uso (y difusión) de los conocimientos sobre las tecnologías avanzadas de construcción.

*Referencia a la factibilidad de construcción en los documentos contractuales.

* Los esfuerzos para realizar un seguimiento de los ahorros u otros efectos de los esfuerzos de constructibilidad.

\section{Puesta en Marcha}

Para implementar con éxito la constructibilidad, el cliente debe dejar claros los objetivos prioritarios del proyecto y permitir que la constructibilidad sea valorada como un atributo del rendimiento del mismo.

\section{Barreras Comunes de la Constructibilidad}

*Objetivos de diseño mal dirigidos.

*Percepción del propietario de lo que hacemos.

*Falta de compromiso genuino con la constructibilidad.

* La falta de comprensión del diseñador de los conceptos de constructibilidad.

*Las habilidades de comunicación entre los constructores son pobres.

* La falta de documentación y recuperación de lecciones aprendidas.

* La falta de formación de equipos o de la asociación.

*Pobre puntualidad de la entrada de constructor.

*Las personas adecuadas no están disponibles.

* Interruptores de barreras fácilmente implementables.

* La renuencia a inversiones adicionales dinero y esfuerzo en el proyecto inicial etapas.

*Falta de experiencia en la construcción del diseño de la organización.

*La falta de mutua del respeto entre diseñadores y constructores.

\section{Destino de los Objetivos de la Constructibilidad}

[7] señalan que los objetivos de la constructibilidad no está orientado únicamente a la facilidad de construcción del proyecto, sino que intenta ser un sistema por el cual se busca la facilidad constructiva y la calidad del producto resultante en las decisiones acerca de la ejecución de la obra, como respuesta a los factores que influyen en el proyecto (tanto exógenos como endógenos) y los objetivos del mismo. Dichos objetivos suelen ser proyectos específicos y de alto valor, como por ejemplo el ahorro de costos, porcentaje de horas de trabajo de ahorro, ahorros en dólares, la reducción por ciento de repetición del trabajo, y la eliminación de los retrasos causados por problemas de contratación. La Industria de la Construcción ha respaldado el potencial de ahorro de costos de los esfuerzos de constructibilidad, el uso óptimo de los conocimientos y experiencia en la construcción, las operaciones de planificación, diseño y sobre el terreno para alcanzar los objetivos generales del proyecto.

\section{Hoja de ruta hito 2}

Establecer un programa de Constructibilidad Corporativa: Este pasó aborda las medidas necesarias para establecer una edificabilidad a nivel corporativo programa, ya sea dueño, diseñador, o las organizaciones de los contratistas. Las funciones del programa y los sistemas de información de apoyo se discuten en detalle. La función principal del patrocinador es mantener un alto nivel del conocimiento y la visibilidad de la constructibilidad programa, El patrocinador debe ser un líder reconocido, que posee las calificaciones adecuadas, debe tener el poder por el poder ejecutivo de gestión de equipos.

*El patrocinador debe ser responsable por el éxito del programa.

* La organización de apoyo proporciona la coordinación e información para facilitar a nivel de proyecto constructibilidad de ejecución.

*Un archivo de las lecciones aprendidas o base de datos se debe establecer para asegurar la mejora continúa de los proyectos.

* Las ideas de factibilidad de construcción se obtienen de muchas diferentes fuentes de una variedad de mecanismos; una forma estándar ayuda en la documentación de las ideas recogidas.

* Las ideas deben ser evaluados para actuales y futuros aplicabilidad, con retroalimentación a los originadores.

* Las ideas seleccionadas deben organizarse y mantenerse en un sistema que faciliten la recuperación futura.

\section{Pasos para un programa de Constructibilidad Cor- porativa}

Pasó 1: Identificar Patrocinador Constructibilidad / Campeón: Los programas exitosos de factibilidad de construcción requieren el apoyo activo de un patrocinador cuya función principal es mantener un alto nivel de conocimiento y la visibilidad del programa de constructibilidad.

El patrocinador del programa, debe ser un líder reconocido dentro de la organización, que posee una amplia experiencia de gestión de proyectos y ser capaz de asegurar que una adecuada aplicación al esfuerzo.

Pasó 2: Establecer la Organización de Apoyo funcional y Procedimientos: según [8] La Coordinación de proyecto a proyecto debe estar garantizada y tener una eficaz vinculación con la información necesaria y la experiencia. El objetivo del 
personal de otros programas y elementos de apoyo es facilitar la aplicación de constructibilidad a nivel de proyecto.

Para lograr esto, otros dos importantes papeles se recomienda que el programa desarrolle:

Un programa responsable de la gerente: Este director del programa, al igual que el patrocinador del programa y la factibilidad de construcción basado en proyectos coordinadores, debe tener la construcción de una gran experiencia y gestión de proyectos, un de trabajo el conocimiento de las necesidades y requisitos de diseño y gran capacidad de comunicación.

Un custodio de base de datos que es responsable de la documentación: El custodio de la base de datos puede también ayuda a los coordinadores del proyecto y el director del programa junto con otros técnicos especialistas en el cribado o la evaluación de las ideas de constructibilidad. Además de la construcción y la experiencia de gestión de proyectos, esta persona debe poseer una fuerte habilidades de comunicación y tienen un conocimiento práctico de manejo de datos computarizados, almacenamiento y recuperación.

Pasó 3: Desarrollar y mantener una base de datos de lecciones aprendidas: La factibilidad de construcción de lecciones aprendidas de base de datos es uno de los tres mecanismos para obtener y documentar las ideas de constructibilidad. Las ideas también pueden provenir de las discusiones del proyecto de las aplicaciones del concepto de factibilidad de construcción y de un programa de sugerencias de constructibilidad, (ya sea corporativa o basado en proyectos).

* La necesidad de una base de datos de lecciones aprendidas no se puede exagerar. Con tal base de datos los fracasos del pasado y los éxitos se pueden grabar en beneficio de los proyectos futuros, lo que refuerza proyecto de mejora continua.

* Las ideas de factibilidad de construcción pueden y debe ser obtenida de diversas fuentes por una variedad de mecanismos. Las fuentes potenciales incluyen a ingenieros, el personal de adquisiciones, superintendentes, capataces, artesanos, vendedores, inspectores, coordinadores de área, personal del propietario del campo y otros de todas las fases del ciclo del proyecto.

* Las ideas de factibilidad de construcción incluyen los informes mensuales sobre la marcha del proyecto, informes finales (liquidación) y personales entrevistas, programas de empleo de sugerencias de sitios de caja, y las actas o notas de las reuniones de planificación del proyecto.

\section{Hoja de ruta hito 3}

Obtener las capacidades de constructibilidad: Para obtener las capacidades de factibilidad de construcción para el proyecto, el propietario debe evaluar a disposición de la casa experiencia y considerar la contratación de diseño externo y la experiencia de constructibilidad.

Cuatro pasos generales conducen a la obtención de las capacidades de factibilidad de construcción:

1. Montar los principales miembros del equipo titular: El gerente del propietario del proyecto y miembros del equipo tienen una influencia decisiva en el éxito del proyecto de factibilidad de construcción.

2. Definir los objetivos y las medidas de factibilidad de construcción: Una vez que los miembros clave de los propietarios del equipo son seleccionados, el equipo puede definir su factibilidad de construcción objetivos y medidas.

3. Seleccione estrategia del proyecto de contratación: La selección de la estrategia de contrato tiene un profundo impacto en el tiempo y la aplicación de constructibilidad de entrada.

4. Los contratistas, vendedores de seguros y consultores: Una vez que el propietario se ha seleccionado la estrategia de contacto para ser utilizado en el proyecto, el siguiente pasó es determinar la forma de facilitar la pronta ejecución del proceso de constructibilidad.

\section{Hoja de ruta hito 4}

Plan de Implementación Constructibilidad: cuatro pasos conducen a la planificación para la implementación efectiva de constructibilidad:

Pasó 1: Desarrollar el equipo de construcción y la identificación / frente a los obstáculos: El equipo integrado por personal de constructibilidad del dueño, diseñador, constructor y de las organizaciones.

También se incluyen representantes de los subcontratistas, proveedores y consultores según sea el caso. A fin de garantizar los intereses mutuos y reducir al mínimo las relaciones antagónicas, los siguientes cuatro tareas deben llevarse a cabo independientemente del tamaño del proyecto.

Es esencial que los individuos clave seleccionados para dirigir los esfuerzos de factibilidad de construcción deban tener experiencia en la construcción, fuertes habilidades interpersonales, y tener la mente abierta. En la selección de los miembros clave de la constructibilidad equipo, el director del proyecto debe considerar los siguientes principios.

*Plan de principios. La planificación puede ayudar a superar barreras como la justificación de los gastos para contratar a personal de la construcción antes de lo que los enfoques tradicionales de gestión de proyectos o no contar con personal disponible apropiadas cuando sea necesario. 
* Insistir en que los individuos con experiencia. Es esencial contar con personal con experiencia del contratista participar en la constructibilidad. Los criterios de selección deben estar señalados con antelación.

* Seleccione los jugadores del equipo de cooperación. Los principales miembros del equipo deben ser capaces de discutir abiertamente problemas con otros participantes del proyecto.

* Minimizar el equipo del proyecto turn-over. La importancia de la continuidad en el equipo del proyecto es esencial para lograr el éxito. Reducción al mínimo de equipo de volumen de negocio aumenta la probabilidad de constructibilidad éxito.

Pasó 2: Pantalla de la base de datos - lecciones aprendidas:

Los equipos de factibilidad de construcción son más eficaces cuando se puede fácilmente aprovechar las lecciones y las innovaciones experimentadas en proyectos anteriores, una base de datos de lecciones aprendidas puede ser un ingrediente clave para la constructibilidad éxito.

Una lista de ejemplos de las lecciones aprendidas se presenta a continuación:

* Plan de vías de acceso para las grúas que se utilizarán para la construcción y mantenimiento.

* Maximizar la construcción a nivel del suelo.

* Planos de cimentación sin problema los detalles de anclaje en negrita cuando el equipo es tal que anclas pueden ser perforados en el después de que el equipo llega.

* Proporcionar cáncamos en pozos de registro prefabricados.

* Diseñar y erecto escaleras permanentes, plataformas y escaleras de mano tan pronto como sea posible.

* Minimizar el uso de aparatos ortopédicos y miembros de los pequeños tanto como sea posible.

* Proporcionar criterios de taller y campo de inspección para todos los equipos de proceso.

Pasó 3: Llevar a cabo el Taller de Planificación Constructibilidad: Preparar y llevar a cabo el Taller de Planificación. El Taller debe llevarse a cabo con todos los actores clave del proyecto y miembros del equipo presentes. Horario-sabio, el taller debe realizarse lo más pronto posible después del análisis de viabilidad del proyecto y el alcance se completa y los participantes del proyecto una vez que son críticos "a bordo".

En la planificación para el Taller de Planificación Constructibilidad, algunos pueden encontrar los conceptos de constructibilidad
Pasó 4: Finalizar los planes de concepto de aplicación: Como se demuestra en los planes de concepto de aplicación, las actividades de factibilidad de construcción deben ser planificados para su aplicación durante las fases de planificación conceptual, diseño y construcción de proyectos. Dos tareas específicas en este pasó se describen a continuación.

Acotar las funciones de constructibilidad, responsabilidades y planes de acción. En la forma de aplicación Concepto planes, un conjunto completo de un enfoque de concepto de acción de los planes es crucial para el éxito de la constructibilidad equipo. Proyecto de factibilidad de construcción a nivel de programa los procedimientos también deben estar preparados. En la preparación de estos procedimientos, el programa de constructibilidad debe basarse en una visión de futuro, la planificación integrada la filosofía en lugar de una retrospectiva "revisión" de diseño completo. Esto minimizará el diseño de trabajo y el resultado en el diseño de mayor calidad.

\section{Herramientas de aplicación de constructibilidad}

\section{Implementación de herramientas descripción del programa}

A continuación se enumeran algunas herramientas fáciles de entender, que se han discutido en otra parte de este documento:

\section{Planificación, Constructibilidad, Agenda del Taller}

1. Presentaciones; instrucciones de seguridad, revisión de la Agenda.

2. Proyecto de Informe de Estado.

3. Resultados / resumen de la orientación de construcción y la evaluación de los obstáculos para aplicación.

4. Mini-grupo de acción Sesiones: Identificación de problemas de constructibilidad y oportunidades.

5. Informes de las inquietudes y oportunidades de quiebre-las sesiones en

6. Priorización de los conceptos de construcción y la sub-conceptos.

7. Los resultados de la preselección de la base de datos Las lecciones aprendidas, los planes para una mayor proyección 8. Mini-grupo de acción Sesiones: Desarrollo de planes de aplicación de conceptos.

9. Los informes de la aplicación de conceptos Planes de ruptura a cabo sesiones.

10. Camino hacia adelante plan de implementación de la aplicación de conceptos Planes.

11. Taller de Cierre.

\section{Requisitos de Organización}

El contratista de la construcción (o gerente de construcción) deberá identificar a una persona en su proyecto equipo para ser responsable de la gestión del programa de constructibilidad del proyecto. Esta persona se considera un individuo clave por el propietario y se comprometan con el proyecto durante la 
duración del proyecto constructibilidad programa. Se reconoce que, basándose en el alcance de la constructibilidad proyecto programa y la fase del proyecto, la participación de tiempo completo por este individuo clave puede no ser necesaria.

En su caso, personal con conocimientos especializados de la construcción será utilizada por el proyecto equipo para proporcionar la entrada de constructibilidad en el tratamiento de temas específicos. El contratista de la construcción (o gerente de construcción) es un miembro clave del equipo titular, que incluye también el arquitecto-ingeniero (y otros miembros, en su caso). Con el fin de cumplido los objetivos de los propietarios en relación con la constructibilidad, que el trabajo en equipo y la integración énfasis total del proyecto, son esencial para el contratista de la construcción (o gerente de construcción) para seleccionar los miembros de su constructibilidad equipo que puede trabajar con eficacia en un entorno de trabajo en equipo.

Reporte: Un registro se mantendrá el cual se enumeran los problemas de factibilidad de construcción que se han considerado, la reducción de costos. Alcanzado (cuando se identifica) y los impactos de horario. Según la definición de los procedimientos del proyecto, un informe periódico se presentará al propietario que muestra estos resultados.

\section{Consideraciones finales}

La gestión de proyectos se desarrolla dentro y fuera de las empresas o comerciales convencionales, donde las actividades son percibidas de forma repetitiva y continua; la gestión de proyectos ha crecido como campo de conocimientos en el mundo de la construcción, allí, ha sido de interés de muchos investigadores por desarrollar la gestión de procesos estables o repetitivos, obras públicas y ejecución de proyectos de I+D. pero aun falta que muchas organizaciones implanten en sus procesos organizacionales la gestión de proyectos.

Desde el punto de vista administrativo lo ideal es que toda organización disponga de alguna forma de descripción de su funcionamiento, que sea fácil de entender tanto para un directivo como para los empleados de menor rango y aun para gente ajena a la organización. Con frecuencia, los organismos que utilizan alguna técnica para describir su funcionamiento, o bien incluyen demasiados detalles, o no están actualizados.

La hoja de ruta de aplicación de la constructibilidaddicha pretende ser útil para los propietarios, diseñadores y contratistas, es dirigida a programas corporativos de constructibilidad y al programa del proyecto de factibilidad de construcción. De igual forma, el análisis de la constructibilidad es mucho mejor sobre la base de la hoja de ruta de aplicación ya que la empresa inicia sus funciones corrigiendo errores desde el inicio. El estudio de la hoja de ruta de aplicación permitir contar con una a guía más fácil para ser implementado en casos donde quieran ser implantados.

\section{Referencias}

[1] SERES M (2006), Gestión Integrada de Proyecto. Politext 96. Ediciones UPC.

[2] FIGUEROA G (2005), La metodología de elaboración de proyectos como una herramienta para el desarrollo cultura. Serie Bibliotecología y Gestión de Información N ${ }^{\mathrm{o}} 7$, septiembre 2005. Revista bajo el ISSN 0718-1701. Chile.

[3] RODRÍGUEZ D (2006), Metodología de Gestión de Proyectos. Dirigid a la diagnostica organizacional. Ediciones Univ ersidad Católica de Chile. Facultad de Ciencias Sociales.

[4] SANTIBÁÑEZ A. (2006), Dirección de Proyectos. Revista de la Universidad Nacional Mayor de San Marcos. Universidad del Perú. Decana de América.

[5] GGPIC: Guías de Gerencia para Proyectos de Inversión de Capital, Revisión 1, Aprobado por: Comité de Operaciones de PDVSA. Caracas Venezuela, 1999

[6] ROBBINS S. (2005), Administración. Editorial Pearson Educación. Octava edición. México.

[7] ROVIRA J., SERES M. Y FREIXA J. "Sert: De la Ciutat Funcional Al Disseny Urbā". Volumen 1 de Dèdal, Volumen 96 de Politext: Organización de empresas, Edicions de la Universitat Politècnica de Catalunya. Barcelona- España, 2006.

[8] Y.C. UREÑA-VILLAMIZAR, Y.C. CASTRILLÓN-JAIMES Y N.Y. CARRUYO-DURÁN, "Respondality: Strategy of the Management in Projects", Respuestas, vol. 23, no. S1, pp. 109 - 112, 2018. doi:10.22463/0122820X.1323 